\title{
Bioanalysis
}

\section{Are we ready for the evolution of electronic laboratory notebooks in regulated bioanalysis?}

\author{
"...electronic laboratory notebook is a great tool for capturing lab \\ workflows and experiments while ensuring data integrity with \\ minimum efforts."
}

First draft submitted: 19 May 2017; Accepted for publication: 15 June 2017; Published online: 2 August 2017

Keywords: computer systems $\bullet$ data integrity $\bullet$ electronic laboratory notebook $\bullet$ validation

During the last decade, quite a few bioanalytical laboratories have adopted electronic laboratory notebooks (ELN) in nonregulated and regulated settings [1-3].

The implementation of an ELN in a regulated bioanalysis laboratory requires a significant amount of resources in order to ensure that the design is truly supporting the internal processes of the lab and is validated in compliance with current regulations.

So, what is preventing a bioanalytical laboratory from implementing an ELN? Other than cost, there is really no significant obstacle anymore to fully adopt electronic record keeping due to the fact that both ELN vendors and laboratories gained sufficient expertise in the implementation of these computerized systems [4,5]. The advantages have become very obvious. In a regulated organization, compliance is usually achieved through procedural controls which are still very prone to human error. This can only be prevented through thorough training and building experience. The implementation of an ELN allows the support of procedural controls through more technical controls which can prevent human errors.

Therefore, the industry is ready for this evolution, as long as the following key aspects in the implementation of a computerized system like ELN are recognized.
As with any computerized system in a regulated organization, the success of an ELN starts with the design. Applying the common $\mathrm{V}$-model or life cycle development model for the implementation of a system [6,7]; the design is critical to ensure that the system can support the internal lab processes in an efficient manner and also ensure data integrity to comply with regulations. Regarding the processes, it is essential that workflow templates within the system are intuitive to use and enable adherence to existing lab procedures. With respect to data integrity, functional requirement specifications in the validation master plan need to clearly define elements such as:

- Roles and security measures;

- Electronic signatures;

- Error prevention;

- Audit trail and log;

- Version control;

- Archiving.

In the following paragraphs, we will briefly describe how these requirements ensure data integrity in electronic laboratory notebooks.

\section{Roles \& security measures}

Roles and security measures provide the access control and limit actions a user can

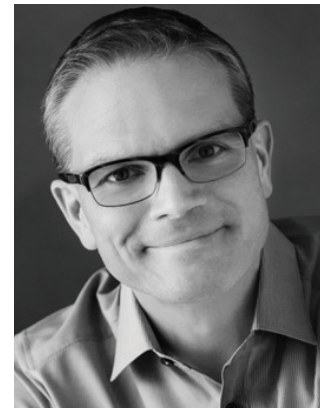

Jens Sydor

DMPK \& Bioanalysis, AbbVie, North Chicago, IL, USA

jens.sydor@abbvie.com

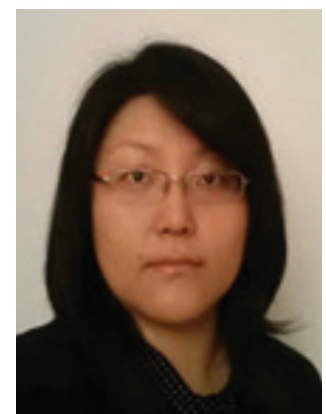

Grace Kim

DMPK \& Bioanalysis, AbbVie, North Chicago, IL, USA newlands press part of 
perform within the system. The system must allow multiple roles to be designed or configured based on the lab workflow and also provide an easy access to retrieve a list of users with associated privileges. Based on the lab process, a group of analysts is allowed to create and edit experiments, but not delete. Yet, another group, for example, Quality Assurance, is configured to only view experiments.

\section{Electronic signature}

Electronic signature is a well-known regulatory requirement [8]. The system must allow users the ability to sign experiments or records by providing username or unique identifier, password and reason or meaning of signature. Also, the system must not allow the copy, transfer or deletion of an electronic signature.

\section{"Due to audit trails at the system and data level, documentation of timely addition of data enables a contemporaneous capture of information."}

\section{Error prevention}

ELN not only replaced the paper documentation but it took one step further. It has enabled the lab workflows and requirements to be incorporated into a system design, instituting a warning system and minimizing human errors. For example, when preparing calibration standards (STD) and quality control (QC) samples, an analyst must ensure stock solutions are not expired and QC concentrations are within certain limits of STD concentrations. The system can be designed to either warn the user when an expired stock solution is used or search only for nonexpired stock solutions for use. If low QC concentrations are not within one-time to three-times of the low STD required as per the standard operating procedure, the system can also warn the user and require the user to correct the error or provide a reason for the deviation. These types of designs allow users to prevent errors immediately without involving another reviewer and prevent errors to be propagated to the next steps. When the system is intelligently designed and validated to reflect the lab processes and regulatory requirements, it will significantly reduce human errors as well as training and review efforts.

\section{Audit trail \& log}

The audit trail is one of the critical pieces in reconstructing an event. It must contain the user ID or unique identifier, description, date/time of event and reason for change. The system must automatically create an audit record on the creation, modification or deletion of data within the system and the system must not allow the modification of an audit record. Since every action by every user is captured, the system should also provide a way to filter hundreds of lines to quickly locate the meaningful information.

\section{Version control}

Along with the audit trails, there is another feature: version control, which provides additional traceability. Every save becomes a new version and the system requires users to provide the username, password and reasons for saving (i.e., electronic signature). This allows users to easily review multiple versions of an experiment or document that may not have the embedded audit trails such as PDF or text file.

\section{Archival}

Last but not least, the computerized system cannot be considered as a whole without addressing data retention and archival. Archival can be addressed procedurally, however, a validated technical solution provides more reliable option. The system must allow archiving of records and limit the access (i.e., readonly). The system can be designed or configured to archive data by logical or physical separation or both. In any case, it is important to ensure that all required metadata such as audit trails and electronic signatures are archived with the data. In addition, both data and metadata must remain unchanged and can be retrieved in human readable format until the data retention period expires.

\section{Data integrity \& ALCOA in ELN}

These and other elements ensure data integrity within the ELN by supporting the ALCOA + principles which require data to be accurate, legible, contemporaneous, original and attributable, as well as complete, consistent, enduring and available when needed [9].

An increase in accuracy can be ensured by the increase in technical controls through error prevention in workflow templates. Legibility is certainly no concern in electronic documentation, significantly improving the reconstruction of old studies even when the documenting analyst left the organization.

Due to audit trails at the system and data level, documentation of timely addition of data enables a contemporaneous capture of information. ELN is the original location where the documentation of a bioanalytical run is captured. However, not all original, raw data can be captured in ELN. Raw data files from analytical equipment are sometimes stored in different locations. Even in this case, ELN can supplement by capturing location of these instruments' raw data as well as their electronic file name in order to allow a 
complete reconstruction of a bioanalytical run. With respect to the last ALCOA attribute, one of the biggest strengths of an audit trail in an ELN is that all entries are clearly attributable to a system user at any time through clear definition of roles and security and electronic signatures.

\section{Conclusion}

Overall, electronic laboratory notebook is a great tool for capturing lab workflows and experiments while ensuring data integrity with minimum efforts. One aspect where both bioanalytical laboratories and regulatory agencies might still struggle at the moment with the evolution of electronic laboratory notebooks is the data availability when needed during inspections. The use of an ELN requires extensive training and experience within a bioanalytical organization. In contrast to paper notebooks, it is much more challenging for inspectors to find the information needed in order to reconstruct studies without knowing the design of the ELN system. Both laboratories and regulators are still learning how to best facilitate inspections in the age of electronic notebooks. In order to facilitate efficient inspections and avoid unnecessary delays, it might be required for bioanalytical laboratories to provide subject matter experts in the front room to assist in the navigation of the system and reconstruct studies.

\section{References}

1 McDowall RD. Developing a strategy for a regulated electronic bioanalytical laboratory. Bioanalysis 6(2), 165-184 (2014).

2 Nussbeck SY, Weil P, Menzel J, Marzec B, Lorberg K, Schwappach B. The laboratory notebook in the 21st century. EMBO Rep. 15(6), 631-634 (2014).

3 Song Y, Dhodda R, Zhang J, Sydor J. A high efficiency, high quality and low cost internal regulated bioanalytical laboratory to support drug development needs. Bioanalysis 6(10), 1295-1309 (2014).

4 Shoup RE, Beato BD, Pisek A et al. Implementation of an electronic laboratory notebook to accelerate data review in bioanalysis. Bioanalysis 5(13), 1677-1689 (2013).

5 Zeng J, Hillman M, Arnold M. Impact of the implementation of a well-designed electronic laboratory

\section{Future perspective}

Even though electronic laboratory notebooks are still not being used in the majority of bioanalytical laboratories, sufficient experience has been gained with these tools to conclude that ELNs can significantly improve data integrity and ensure compliance with internal workflows by minimizing human error. In addition, regulators are more and more focused on understanding the usage of such systems during inspections and increasing their knowledge for data review. Therefore, it can be assumed that electronic laboratory notebooks will be adopted by an increasing number of laboratories in the future, considering the additional capabilities that these electronic notebooks and other new interfacing systems will be able to offer, such as asset management, usage tracking and searching capabilities across multiple records.

\section{Financial \& competing interests disclosure}

The authors have no relevant affiliations or financial involvement with any organization or entity with a financial interest in or financial conflict with the subject matter or materials discussed in the manuscript. This includes employment, consultancies, honoraria, stock ownership or options, expert testimony, grants or patents received or pending, or royalties.

No writing assistance was utilized in the production of this manuscript.

notebook on bioanalytical laboratory function. Bioanalysis 3(13), 1501-1511 (2011).

$6 \quad G A M P^{\circledR}$ 5:A Risk-Based Approach to Compliant GxP Computerized System. International Society for Pharmaceutical Engineering, Bethesda, MD, USA (2008).

7 OECD Series on Principles of Good Laboratory Practice and Compliance Monitoring Number 17: Application of GLP Principles to Computerized Systems. Organization for Economic Co-operation and Development, Paris, France (2016).

8 Guidance for Industry Part 11, Electronic Records; Electronic Signatures - Scope and Application. US FDA, MD, USA (2003).

9 Reflection Paper on Expectations for Electronic Source Data and Data Transcribed to Electronic Data Collection Tools in Clinical Trials. European Medicines Agency, London, UK (2010). 